\title{
A maloclusão do tipo II de Angle pode ser tratada com o uso exclusivo de alça de retração do tipo Bull? Relato de caso clínico
}

Can Angle's type II malocclusion be treated with the exclusive use of a Bull-type retraction loop? Clinical case report

¿Se puede tratar la maloclusión tipo II de Angle con el uso exclusivo de un ansas de retracción tipo

\author{
Bull? Reporte de caso clínico
}

Recebido: 21/03/2021 | Revisado: 26/03/2021 | Aceito: 28/03/2021 | Publicado: 09/04/2021

\author{
Kim Henderson Carmo Ribeiro \\ ORCID: https://orcid.org/0000-0003-3325-5897 \\ Universidade Estadual Paulista, Brasil \\ E-mail: kimhenderson@hotmail.fr \\ Juliana De Aguiar Silveira Meira \\ ORCID: https://orcid.org/0000-0003-2340-2005 \\ Universidade Estadual Paulista, Brasil \\ E-mail: juliana.aguiarmeira@ hotmail.com \\ Ana Paula Simões Corrêa \\ ORCID: https://orcid.org/0000-0002-8207-4606 \\ Universidade Estadual Paulista, Brasil \\ E-mail: paulinha_odonto@hotmail.com \\ Mariza Akemi Matsumoto \\ ORCID: https://orcid.org/0000-0001-5389-0105 \\ Universidade Estadual Paulista, Brasil \\ E-mail: mariza.am@gmail.com \\ Evandro da Silva Bronzi \\ ORCID: https://orcid.org/0000-0002-9907-7125 \\ Instituto Brasileiro de Ensino do Norte, Brasil \\ E-mail: ebronzi@hotmail.com \\ Juan Miguel Antezana Vera \\ ORCID: https://orcid.org/0000-0002-2219-4200 \\ Instituto Brasileiro de Ensino do Norte, Brasil \\ E-mail: juan.miki@hotmail.com
}

\begin{abstract}
Resumo
A má oclusão Classe II de Angle é caracterizada por uma discrepância dentária anteroposterior, que geralmente está acompanhada por alterações esqueléticas. O tratamento ortodôntico tardio, permite a correção desta maloclusão havendo principalmente necessidade de extrações dentárias. Este relato apresenta uma opção de tratamento de classe II de Angle com uso de alça de retração do tipo Bull, abordando as vantagens da técnica e estabelecendo sua eficácia. Paciente do sexo masculino, apresentava perfil facial convexo e foi diagnosticado com classe II de Angle. O tratamento iniciou com colagem do aparelho fixo, e colocação de placa de levante anterior (PLA) modificada com barra transpalatina. Posteriormente, foi realizado a exodontia dos primeiros pré-molares superiores e feita retração do segmento anterior com a alça de retração do tipo Bull Loop até fechamento dos espaços, e relação de caninos em classe I além de boa estabilização oclusal. Por fim, as contenções foram instaladas para preservar as posições dentárias alcançadas. $\mathrm{O}$ paciente apresentou resultados satisfatórios, com todas as guias e funções restauradas além de um perfil harmônico, evidenciando dessa forma a eficácia da técnica abordada.
\end{abstract}

Palavras-chave: Movimentação dentária; Ortodontia; Procedimentos ortodônticos de ancoragem; Classe II de Angle; Maloclusão.

\begin{abstract}
Angle Class II malocclusion is characterized by an anteroposterior dental discrepancy, which is usually accompanied by skeletal changes. The late orthodontic treatment allows the correction of this malocclusion with the need for tooth extractions. This report presents an Angle class II treatment option with the use of a retraction loop of the Bull type, addressing the advantages of the technique and establishing its effectiveness. Male patient, with a convex facial profile was diagnosed with Angle class II. The treatment started with conventional orthodontic brackets, and placement of a modified anterior lift plate with transpalatal bar. Subsequently, extraction of the first maxillary premolars was performed and the anterior segment was retracted using the Bull Loop retraction loop until the spaces were closed, and canine angulation in class I was achieve, in addition to good occlusal stabilization. Finally, a $3 \times 3$ fixed retainer and
\end{abstract}


superior removable braces were installed to preserve the achieved dental positions. The patient presented satisfactory results, with all guides and functions restored, in addition to a harmonic profile, thus showing the effectiveness of the approached technique.

Keywords: Tooth movement; Orthodontics; Orthodontic anchorage procedures; Angle class II; Malocclusion.

\section{Resumen}

La maloclusión de Angle Class II se caracteriza por una discrepancia dental anteroposterior, que suele ir acompañada de cambios esqueléticos. El tratamiento de ortodoncia tardío permite corregir esta maloclusión con la necesidad de extracciones dentales. Este informe presenta una opción de tratamiento de clase II para Angle con el uso de un ansas de retracción del tipo Bull, abordando las ventajas de la técnica y estableciendo su efectividad. Paciente de sexo masculino, de perfil facial convexo y diagnosticado de clase II de Angle. El tratamiento comenzó con la unión del aparato fijo y la colocación de una placa de elevación anterior modificada con una barra transpalatina. Posteriormente se realizó la extracción de los primeros premolares superiores y se retrajo el segmento anterior mediante el ansas de retracción Bull Loop hasta cerrar los espacios, y una relación canina clase I, además de una buena estabilización oclusal. Finalmente, se instalaron los retenedores para preservar las posiciones dentales logradas. El paciente presentó resultados satisfactorios, con todas las guías y funciones restauradas, además de un perfil armónico, mostrando así la efectividad de la técnica abordada.

Palabras clave: Movimiento dentario; Ortodoncia; Procedimientos de anclaje ortodóncico; Ángulo clase II; Maloclusión.

\section{Introdução}

A má oclusão de Classe II, consiste em aproximadamente $40 \%$ da população mundial, e o seu tratamento representa um desafio para os ortodontistas, pois se diferem bastante em relação à etiologia, diagnóstico e plano de tratamento (Silva et al., 1990). O sucesso do tratamento desta má oclusão pode ser significativamente influenciado por fatores como o protocolo de tratamento (Angle, 1928; Bishara et al.,1995), severidade da má oclusão (Bartsch et al., 1993), discrepância ântero-posterior, idade do paciente (Nanda \& Kierl, 1992) e o seu grau de colaboração com o tratamento (Draker, 1960; Jasper \& McNamara, 1995; Dewel, 1955; Janson et al., 2004). Considerando-se os protocolos de tratamento, diversos mecanismos podem ser utilizados para sua correção, como: aparelhos ortopédicos, distalizadores, elásticos intermaxilares de Classe II, extrações de quatro pré-molares ou apenas dois pré-molares superiores, extrações de segundos molares e até mesmo cirurgias ortognáticas, em caso de grandes discrepâncias ósseas (Dewel, 1955; Janson et al., 2004; Shimizu et al., 2002). As extrações de pré-molares, como um meio de tratamento, para aliviar o apinhamento dos dentes anteriores e corrigir a protrusão dentária são recomendadas desde o século XVIII (Souza et al., 2003). O tratamento com extrações de dois primeiros pré-molares superiores deve ser considerado quando o paciente apresenta um bom arco inferior e não possui mais crescimento, e vem se apresentando com maior eficácia do que com quatro extrações ou sem extrações (Bull, 1951; Ferreira et al., 2005).

Vários dispositivos podem ser empregados para se obter a movimentação dentária (Fryar, 1960), podendo-se optar por uma mecânica de deslizamento ao longo de arcos ortodônticos contínuos ou, ainda, por uma mecânica isenta de fricção, onde arcos segmentados com alças ortodônticas podem ser empregados. Como já citado como hipótese de tratamento, as alças de retração continuam sendo muito utilizadas. Uma vez ativadas, as alças exercem carregamento sobre um ou mais dentes e esses o transmitem para o ligamento periodontal, produzindo movimentação dentária. Conforme o plano de tratamento delineado, pode-se obter a retração isolada de caninos, como podem ser utilizadas como componentes dos arcos segmentados para a retração do bloco anterior (Ferreira et al., 2004).

Diversos tipos de alças de retração foram descritos na literatura, quanto as alças de Bull (Bull loop), ela pode ser utilizada em fios contínuos e localizada na distal dos caninos, para a retração do segmento anterior. Em relação a pré-ativação da alça Bull, recomenda-se a realização do efeito Gable para evitar extrusão e inclinações excessivas dos incisivos para a lingual, com aprofundamento da mordida (Kapila \& Sachdeva, 1989).

Neste trabalho abordou-se a retração anterior em massa de um paciente Classe II com alça de retração do tipo Bull, com exodontia dos dois primeiros pré-molares superiores. 


\section{Metodologia}

Este trabalho corresponde a um relato de caso de forma descritiva e qualitativa por meio do uso de arquivo clínico do atendimento de um paciente pela equipe de Ortodontia e Ortopedia Funcional dos maxilares da UNICSUL - Manaus, Amazonas, Brasil, que apresentou deformidade dento-facial do tipo classe II e foi tratado com o uso exclusivo de alça do tipo Bull (Ferreira et al., 2008), sendo autorizado pelo paciente por meio de um Termo de Consentimento Livre e Esclarecido (TCLE) e submetido ao comitê de ética (Plataforma Brasil). Além de detalhar a eficácia e expor as singularidades da abordagem escolhida, comparando com outros tratamentos para a mesma má-oclusão por meio da leitura de artigos científicos nas bases de dados National Library of Medicine (PubMed), Literatura Latino-Americana e do Caribe em Ciências da Saúde (LILACS), Scientific Electronic Library Online (SciELO) e "Google Acadêmico". As palavras chaves foram selecionadas de acordo com os Descritores em Ciências da Saúde (Decs), sendo escolhidas de acordo com a relevância dos assuntos abordados (Pereira et al., 2018).

\section{Relato de Caso}

Paciente do sexo masculino, caucasiano, 24 anos, foi encaminhado a clínica de especialização em ortodontia da unidade avançada de pós-graduação Manaus-AM Universidade Cruzeiro do Sul (UNICSUL) para avaliação ortodôntica. Foi realizada anamnese e avaliação clínica do paciente, onde o paciente relatou ser portador de asma, rinite alérgica, sinusite e possuir respiração predominante nasal além de hábitos, parafuncionais como onicofagia e morder bochechas. Sua queixa principal: "dentes tortos e amarelos".

$\mathrm{Na}$ análise facial frontal (Imagem 1A, B) o paciente apresentava simetria facial, o terço inferior aumentado, padrão mesofacial, linha média coincidente superior e inferior, porém linha média não coincidente com o pogônio. O perfil facial (Imagem 1C) do tipo convexo, ângulo do perfil classe II de Angle, ângulo nasolabial fechado, ponta nariz para baixo, lábio superior mais projetado, sulco geniano profundo, os terços faciais desproporcionais com uma altura facial inferior (AFAI) DE $143 \mathrm{~mm}$, e comprimento queixo-pescoço de $71 \mathrm{~mm}$.

Ao exame clínico intra-oral pode-se visualizar os molares direitos em classe II (Imagem 1D) e o esquerdo classe II completa (Imagem 1E), os caninos apresentavam-se em classe II, leve vestibularização dos incisivos superiores e inferiores, dentadura permanente e sobressaliência acentuada (Imagem 1F). Foi feita manipulação mandibular para correto posicionamento oclusal e observado que a máxima intercuspidação habitual (MIH) e relação cêntrica (R.C) eram coincidentes. 
Imagem 1. Exame clínico inicial. A- Fotografia extra-bucal sorrindo. B- Vista frontal. C- Vista lateral. D- Fotografias intraorais lateral direito. E- Lateral esquerdo. F- Frontal.

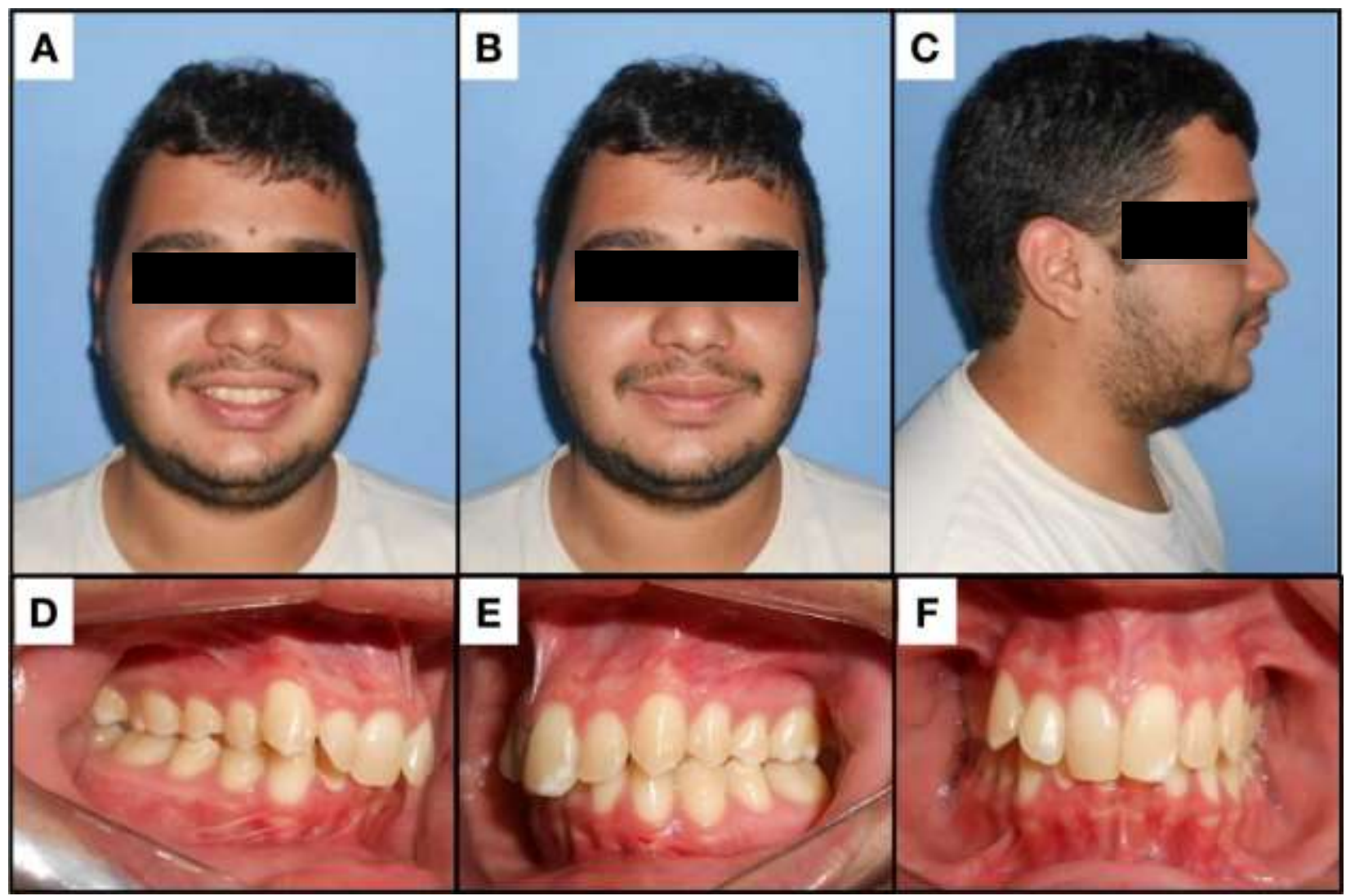

Fonte: Autores.

Ao analisar a radiografia panorâmica (Imagem 2A) solicitada, observou-se todos os elementos dentários permanentes presentes; Elementos dentários 18, 38 e 48 inclusos e 28 fora da linha de oclusão. Na teleradiografia cefalométrica em norma lateral (Imagem 2B), podemos observar leve vestibularização dos incisivos superiores e vestibularização dos inferiores, padrão mesofacial, classe II esquelética e perfil convexo além dos tecidos moles com lábio superior protruído. Em seguida foi realizado o traçado cefalométrico afim de se avaliar a análise cefalométrica como auxílio no tratamento (Imagem 2C), levando em consideração: posição maxila e mandíbula (Tabela 1), relação maxilo-mandibular (Tabela 2), análise do padrão dentário (Tabela 3).

Imagem 2. Exames de imagem inicial. A- Radiografia panorâmica. B- Teleradiografia em norma lateral. C- Traçado cefalométrico inicial.
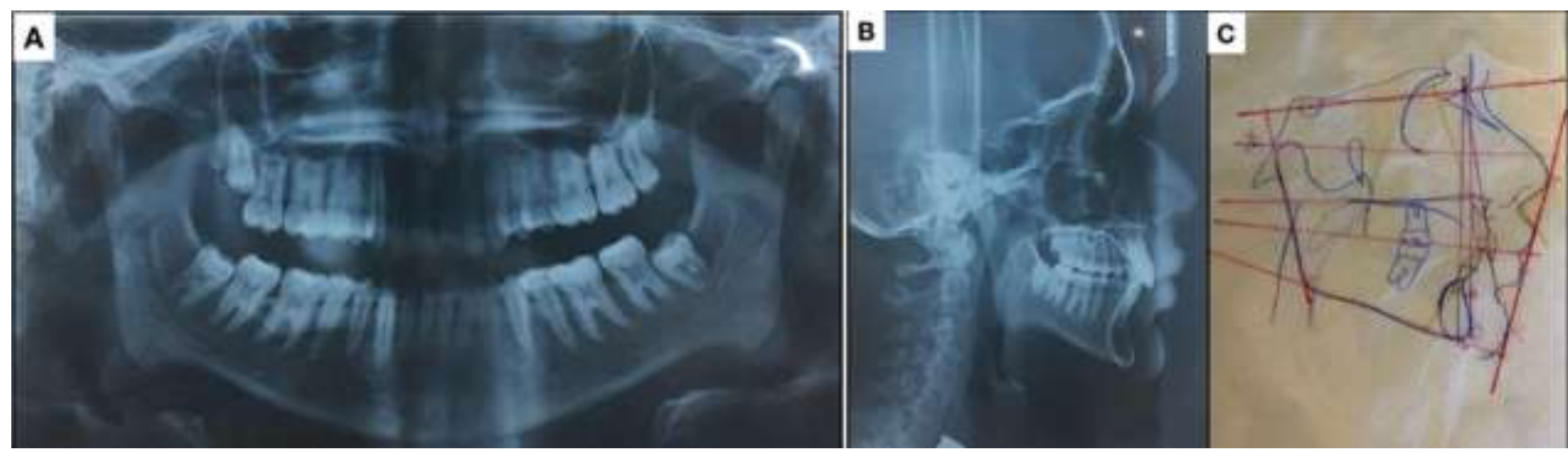

Fonte: Autores. 
Tabela 1. Posição maxila e mandíbula.

\begin{tabular}{|c|c|c|c|}
\hline Medida & Valor Padrão & Valor Medido & Posicionamento \\
\hline SNA & $82^{\circ}+/-2$ & $89^{\circ}$ & Maxila Protruída \\
\hline A-Nperp & $0-2 \mathrm{~mm}$ & $8,5 \mathrm{~mm}$ & Protrusão Maxilar \\
\hline SNB & $80^{\circ}+/-2$ & $78^{\circ}$ & Mandíbula bem \\
\hline Pg-Nperp & $-0,32+/-3$ & & posicionada \\
\hline FNP & $87^{\circ}+/-3$ & $3,2 \mathrm{~mm}$ & Normal \\
& & $91^{\circ}$ & Classe III \\
\hline
\end{tabular}

Fonte: Autores.

Tabela 2. Relação Maxilo-mandibular.

\begin{tabular}{|c|c|c|c|}
\hline Medida & Valor Padrão & Valor Medido & Posicionamento \\
\hline ANB & $2,0+/-2^{\circ}$ & $9,5^{\circ}$ & Padrão esquelético classe \\
\hline WITS & $-0,1+/-2$ & & II \\
\hline Co-A & $100+/-4 \mathrm{~mm}$ & $8,4 \mathrm{~mm}$ & Diminuído \\
\hline Co-Gn & $130+/-5 \mathrm{~mm}$ & $112 \mathrm{~mm}$ & Pequeno \\
\hline
\end{tabular}

Fonte: Autores

Tabela 3. Análise do padrão dentário.

\begin{tabular}{|c|c|c|c|}
\hline Medida & Valor Padrão & Valor Medido & Posicionamento \\
\hline 1.NA & $22^{\circ}$ & $10^{\circ}$ & Horizontal \\
\hline 1-NA & $4 \mathrm{~mm}$ & $0,5 \mathrm{~mm}$ & Retraído \\
\hline $\mathbf{1 - N B}$ & $25^{\circ}$ & $30^{\circ}$ & Vestibularizado \\
\hline Pg-NB/1-NB & $4 \mathrm{~mm}$ & $7,5 \mathrm{~mm}$ & Aumentado \\
\hline $\mathbf{1 . 1}$ & 1 & 0,6 & Normal \\
\hline $\mathbf{1 . S t}$ & $131^{\circ}+/-10$ & $132^{\circ}$ & Normal \\
\hline IMPA & $2,5 \mathrm{~mm}+/-0,5 \mathrm{~mm}$ & $7 \mathrm{~mm}$ & Aumentado \\
\hline
\end{tabular}

Fonte: Autores.

O tratamento se deu em duas etapas. Na primeira fase, foi realizado a colagem do aparelho fixo superior e inferior, prescrição Capalloza Padrão II e colocado a placa de levante anterior (PLA) modificada com barra transpalatina (Imagem 3A) e iniciou-se o alinhamento e nivelamento com fios nitinol, por um período de 7 meses.

Na segunda fase do tratamento ortodôntico, foi realizado a exodontia dos primeiros pré-molares superiores (elementos 14 e 24). A PLA foi removida e manteve-se a barra transpalatina para maior ancoragem posterior, permitindo assim uma retração dos dentes anteriores, com o fio 0,017 ” x 0,025 ” por meio de alça de retração do tipo Bull Loop (Imagem 3B, C) e 
realizado efeito gable no fio, para evitar devolver a sobremordida ao paciente. Mensalmente a alça de retração foi ativada, deixando, após 5 meses, os caninos em chave (Imagem 4A).

Imagem 3. A- Placa de levante anterior com barra transpalatina. B- Confecção da alça de retração do tipo Bull. C- Efeito gable.
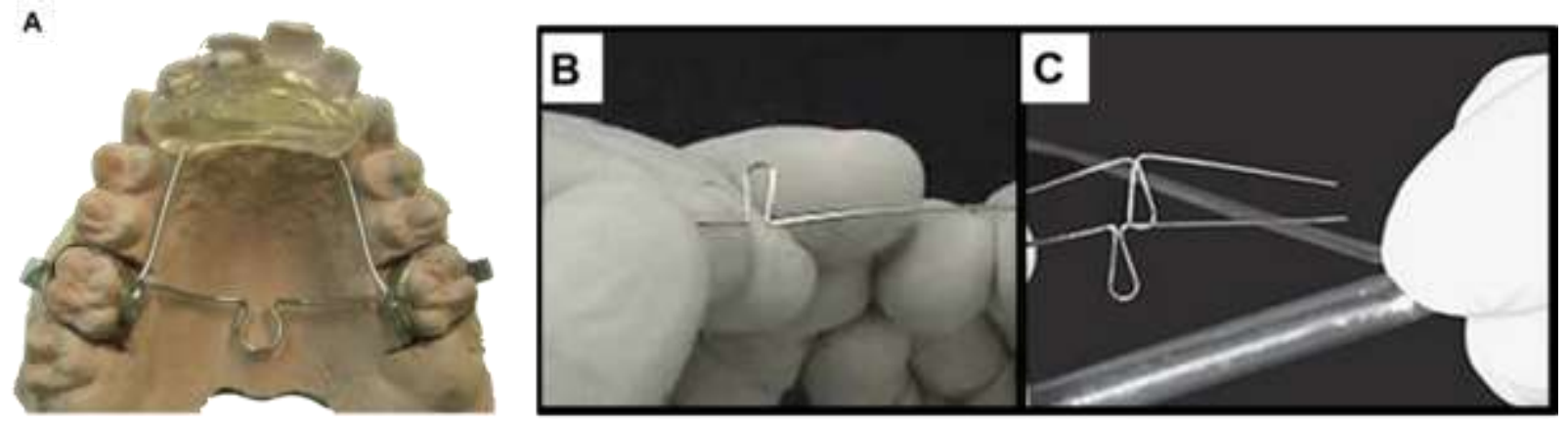

Fonte: Autores.

Foi solicitado uma nova radiografia panorâmica onde observou-se raízes dentárias bem posicionadas (Imagem 5A). Em seguida foi removida a barra transpalatina (Imagem 4B), com perda de ancoragem e colocação de elástico corrente de molar a molar na arcada superior (Imagem 4C) e inferior para fechamento de pequenos diastemas.

Imagem 4 Exame intra-oral após a remoção da alça Bull. A- Fotografia intra-oral caninos em classe I. B- Remoção da barra transpalatina. C- Elástico corrente na arcada superior (vista frontal).

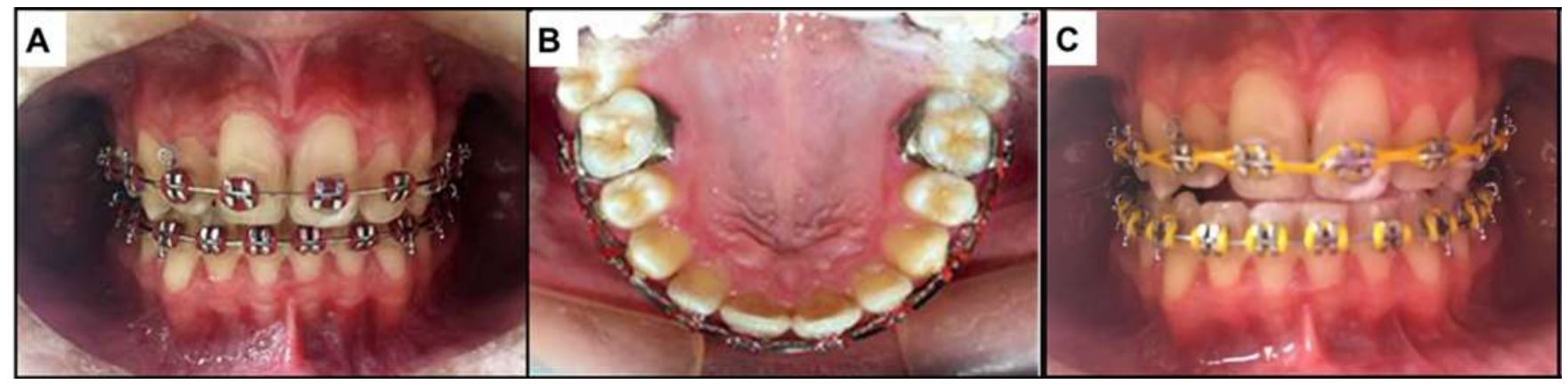

Fonte: Autores.

O paciente fez uso de elásticos para intercuspidação maxilo-mandibular por dois meses. Por fim, uma nova telerradiografia foi solicitada (Imagem 5B) para realização de novos traçados cefalométricos (Imagem 5C). Onde foi possível observar padrões faciais e dentários mais próximos da normalidade e melhora dos posicionamentos dentários, ao compararmos os valores obtidos antes e após o tratamento ortodôntico (Tabela 4). 
Imagem 5. Exames de imagem final. A- Radiografia panorâmica pós-retração. B- Telerradiografia norma lateral. C- Traçado cefalométrico final.
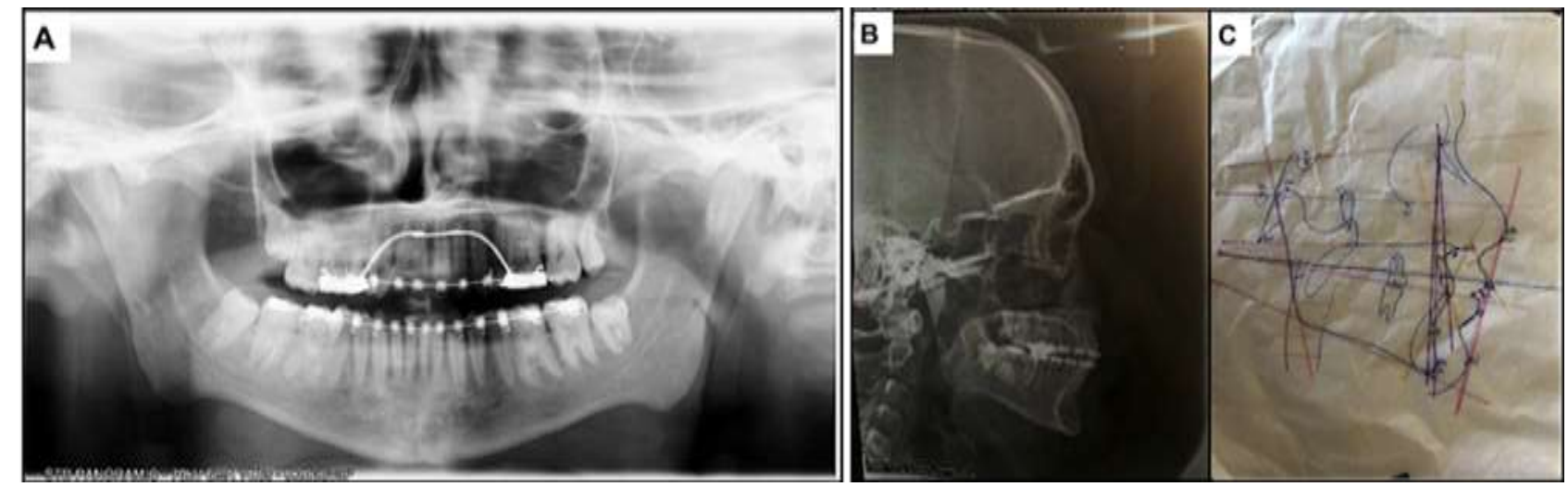

Fonte: Autores.

Tabela 4. Análise dentária antes e depois da ortodontia.

\begin{tabular}{|c|c|c|}
\hline Medida & Valor Padrão & Valor Medido \\
\hline 1.NA & $10^{\circ}$ & $13^{\circ}$ \\
\hline 1-NA & $0,5 \mathrm{~mm}$ & $3 \mathrm{~mm}$ \\
\hline $\mathbf{1}$-NB & $30^{\circ}$ & $24^{\circ}$ \\
\hline Pg-NB/1-NB & $7,5 \mathrm{~mm}$ & $6 \mathrm{~mm}$ \\
\hline $\mathbf{1 . 1}$ & 0,6 & 0,9 \\
\hline $\mathbf{1 . S t}$ & $132^{\circ}$ & $139^{\circ}$ \\
\hline IMPA & $7 \mathrm{~mm}$ & $4 \mathrm{~mm}$ \\
\hline
\end{tabular}

Fonte: Autores.

A aparelhagem fixa superior e inferior foi então removida, o paciente atingiu guia protrusiva anterior e guia canina (Imagem 6A, B), sem interferências, caninos em classe I e todos os elementos dentários em posição sem contatos prematuros e as contenções foram instaladas (Imagem 6C, D, E). No acompanhamento de 4 anos, observa-se estabilidade do caso. 
Imagem 6. Exame intra e extra-oral após o tratamento. A- Vista frontal da guia protusiva anterior e guia canina. B- Vista lateral direita da guia protusiva anterior e canina. C- Contenção superior instalada. D- Contenção inferior. E- Paciente em vista frontal após colocação da contenção.

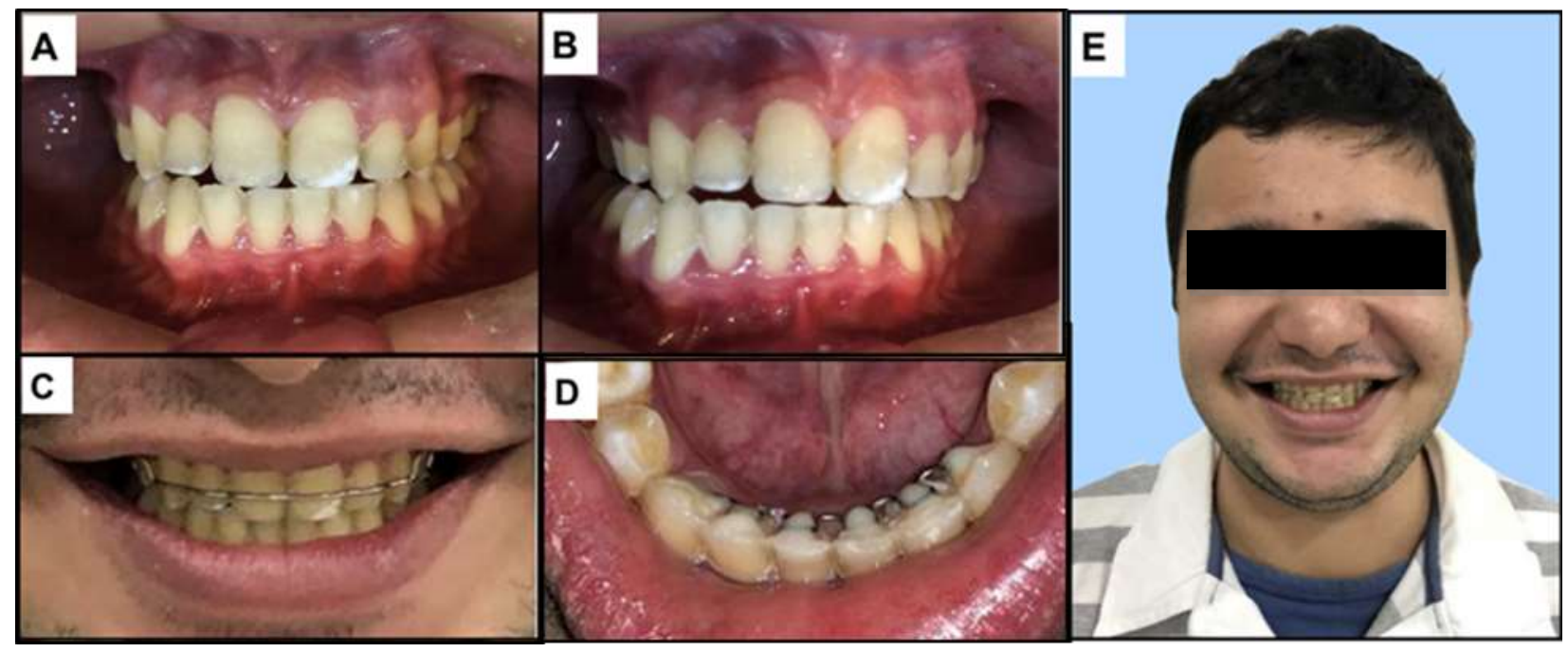

Fonte: Autores.

\section{Resultado e Discussão}

A protusão maxilar é comumente tratada por extração de pré-molares que resultam em oclusão de caninos classe I e de molares classe II, por meio de retração anterior para atingir um perfil facial harmonioso e uma relação adequada dos incisivos. O sistema biomecânico aplicado deve garantir a retração adequada dos dentes anteriores para função, estética e estabilidade (Sadek et al., 2019). Em casos graves de protusão, muitas vezes é necessária máxima ancoragem por meio de dispositivos como barras transpalatinas, utilizada no presente caso (Vaden et al., 2018). Esses auxiliares ajudam a alcançar resultados aceitáveis para reduzir overjet quando se tem uma boa cooperação do paciente (Kuroda et al., 2009)

Autores citam que na maioria dos casos limítrofes, os pacientes apresentam um padrão esquelético adequado e aceitável, e equilíbrio adequado do tecido mole, uma condição que frequentemente é indicada para extração em $5 \%$ a $87 \%$ dos casos. Em qualquer má oclusão, e particularmente em um caso limítrofe, é necessário avaliar as características dentais, faciais e esqueléticas do paciente para estabelecer um diagnóstico correto e um eficaz plano de tratamento (Ruellas et al., 2010). Nossa escolha de extração dos primeiros pré-molares superiores, foi justificada por um perfil convexo do paciente, permitindo assim, uma mecânica de retração total anterior, finalizando com um perfil mais reto e harmônico. Esses dentes são geralmente escolhidos por causa da sua posição e tamanho, que são compatíveis com a maioria dos tipos de discrepâncias nos casos que requerem a retração de dentes anteriores (Araújo \& Caldas, 2019).

Diversas alças de retração ortodôntica, com finalidade de fechar espaços de maneira sistemática e controlada, foram estudadas e desenvolvidas ao longo dos anos. Como exemplo, as clássicas alças retangulares verticais, com loop fechado, desenvolvidas por Bull (Ferreira et al., 2008) tal qual relatado. A principal vantagem da mecânica de loop é a falta de atrito entre o braquete e o fio do arco durante o fechamento do espaço. No entanto associada a esta técnica estão as rotações indesejáveis dos dentes nos planos transversais e sagitais que pode ser minimizado por dobras e torques nos fios, como o efeito Gable, aqui utilizado (Sanjay et al., 2015).

Estudos mostram que o uso de barra palatina e fechamento de espaço em duas fases são as duas condutas mais utilizadas para ancoragem posterior (Monini et al., 2013), que corraboram com o nosso protocolo aplicado. Algumas situações requerem algumas técnicas em detrimento de outras, e o ortodontista pode ter suas próprias preferências. Independentemente do método a ser utilizado, um bom conhecimento da biomecânica é essencial (Ribeiro \& Jacob, 2016). 


\section{Conclusão}

O uso exclusivo de alça do tipo Bull com efeito Gable para tratar a classe II do paciente apresentou resultados satisfatórios, obtendo o fechamento de espaço total, diminuição do trespasse horizontal e permitindo guias caninas e incisivas adequadas.

O aparelho ortodôntico mostrou-se eficiente na fase de alinhamento e nivelamento, corrigindo posicionamentos dentários, assim como intrusões dos incisivos superiores. Enquanto a placa de levante anterior modificada com barra transpalatina, mostrou-se de grande importância para corrigir a sobremordida e dar maior ancoragem posterior.

O final do tratamento mostrou que o objetivo proposto foi alcançado, uma vez que, o paciente saiu com todas as guias e funções restauradas e satisfeito com a opção de tratamento.

Apesar do resultado positivo nesse caso, sugerimos mais estudos utilizando exclusivamente alças de retração para a maloclusão em questão.

\section{Referências}

Angle, E. H. (1928). The Latest and Best in Orthodontic Mechanism. The Dental cosmos, 70 (12), 1143-1158.

Araújo, T. M. \& Caldas, L. D. (2019). Tooth extractions in Orthodontics: first or second premolars? Dental press journal of orthodontics, 24 (3), 88-98. https://doi.org/10.1590/2177-6709.24.3.088-098.bbo

Bartsch, A., Witt, E., Dietz, I. \& Dietz, P. (1993). The clinical and psychological indicators of behavior in wearing a removable appliance. Fortschritte der Kieferorthopadie, 54 (3), 119-128. 10.1007/bf02409021

Bishara, S. E., Cummins, D. M. \& Jakobsen, J. R. (1995). The morphologic basis for the extraction decision in Class II, division 1 malocclusions: a comparative study. American journal of orthodontics and dentofacial orthopedics: official publication of the American Association of Orthodontists, its constituent societies, and the American Board of Orthodontics, 107 (2), 129-135. https://doi.org/10.1016/s0889-5406(95)70127-3

Bull, H. L. (1951). Obtaining facial balance in the treatment of Class II, division 1. Angle Orthod. Appleton, 21 (3), $139-149$.

Dewel, B. F. (1955). Second premolar extraction in orthodontics: principles procedures and case analysis. Am J Orthod, 41 (2), $107-120$.

Draker, H. L. (1960). Handicapping labio-lingual deviations: A proposed index for public health purposes. American Journal of Orthodontics, 46 (4), 295-305. https://doi.org/10.1016/0002-9416(60)90197-4

Ferreira, M. A., Ignácio, A. S., Luersen, M. A. \& Borges, P. C. (2004). Efeito da secção transversal num protótipo de alça de retração ortodôntica de geometria complexa pelo método dos elementos finitos. Rev. de Clín. Pesq. Odontol., 1 (2).

Ferreira, M. A., de Oliveira, F. T., Ignácio, A. S. \& Borges, P. C. (2005). Experimental force definition system for a new orthodontic retraction spring. The Angle orthodontist, 75 (3), 368-377. https://doi.org/10.1043/0003-3219(2005)75[368:EFDSFA]2.0.CO;2

Ferreira, M. A., Borges, P. C. \& Luersen, M. A. (2008). Alguns aspectos da mecânica das alças de retração ortodôntica. Revista Dental Press de Ortodontia e Ortopedia Facial, 13 (3), 112-123. https://doi.org/10.1590/S1415-54192008000300014

Fryar, G. M. (1960). Load deflection determinations of specific wire configurations. [dissertação de mestrado]. Indianapolis, Ind: University of Indiana.

Janson, G., Brambilla, A., Henriques, J. F., de Freitas, M. R. \& Neves, L. S. (2004). Class II treatment success rate in 2- and 4-premolar extraction protocols. American journal of orthodontics and dentofacial orthopedics: official publication of the American Association of Orthodontists, its constituent societies, and the American Board of Orthodontics, 125 (4), 472-479. https://doi.org/10.1016/j.ajodo.2003.04.013

Jasper, J. J. \& McNamara, J. A. (1995). The correction of interarch malocclusions using a fixed force module. American journal of orthodontics and dentofacial orthopedics: official publication of the American Association of Orthodontists, its constituent societies, and the American Board of Orthodontics, 108 (6), 641-650. https://doi.org/10.1016/s0889-5406(95)70010-2

Kapila, S. \& Sachdeva, R. (1989). Mechanical properties and clinical applications of orthodontic wires. American journal of orthodontics and dentofacial orthopedics: official publication of the American Association of Orthodontists, its constituent societies, and the American Board of Orthodontics, 96 (2), 100109. https://doi.org/10.1016/0889-5406(89)90251-5

Kuroda, S., Yamada, K., Deguchi, T., Kyung, H. M. \& Takano-Yamamoto, T. (2009). Class II malocclusion treated with miniscrew anchorage: comparison with traditional orthodontic mechanics outcomes. American journal of orthodontics and dentofacial orthopedics: official publication of the American Association of Orthodontists, its constituent societies, and the American Board of Orthodontics, 135 (3), 302-309. https://doi.org/10.1016/j.ajodo.2007.03.038

Monini, A. C., Gandini Júnior, L. G., Santos-Pinto, A., Maia, L. G. M. \& Rodrigues, W. C. (2013). Procedures adopted by orthodontists for space closure and anchorage control. Dental Press Journal of Orthodontics, 18 (6), 86-92. https://dx.doi.org/10.1590/S2176-94512013000600013

Nanda, R. S. \& Kierl, M. J. (1992). Prediction of cooperation in orthodontic treatment. American journal of orthodontics and dentofacial orthopedics: official publication of the American Association of Orthodontists, its constituent societies, and the American Board of Orthodontics, 102 (1), 15-21. https://doi.org/10.1016/0889-5406(92)70010-8 
Research, Society and Development, v. 10, n. 4, e24010414107, 2021

(CC BY 4.0) | ISSN 2525-3409 | DOI: http://dx.doi.org/10.33448/rsd-v10i4.14107

Pereira, A. S., Shitsuka, D. M., Parreira, F. J. \& Shitsuka, R. (2018). Metodologia da pesquisa científica. Santa Maria, RS: UAB/NTE/UFSM. https://repositorio.ufsm.br/bitstream/handle/1/15824/Lic_Computacao_Metodologia-Pesquisa-Cientifica.pdf?sequence=1

Ribeiro, G. L. U. \& Jacob, H. B. (2016). Understanding the basis of space closure in Orthodontics for a more efficient orthodontic treatment. Dental Press Journal of Orthodontics, 21 (2), 115-125. https://doi.org/10.1590/2177-6709.21.2.115-125.sar

Ruellas, A. C. O., Ruellas, R. M. O., Romano, F. L., Pithon, M. M. \& Lacerda-Santos, R. (2010). Extrações dentárias em Ortodontia: avaliação de elementos de diagnóstico. Dental Press Journal of Orthodontics. https://doi.org/10.1590/S2176-94512010000300017

Sadek, M. M., Sabet, N. E. \& Hassan, I. T. (2019). Type of tooth movement during en masse retraction of the maxillary anterior teeth using labial versus lingual biocreative therapy in adults: A randomized clinical trial. Korean journal of orthodontics, 49 (6), 381-392. https://doi.org/10.4041/kjod.2019.49.6.381

Sanjay, N., Rajesh, R. N., Scindia, R. \& Ajith, S. D. (2015). Space closure with loop mechanics for treatment of bimaxillary protrusion: a case report. Journal of international oral health: JIOH, 7 (5), 65-67.

Shimizu R. H., Sakima T., Pinto A.S. \& Shimizu I.A. (2002) Desempenho biomecânico da alça em "T" construída com fio de aço-inoxidável, durante o fechamento de espaços no tratamento ortodôntico. Rev Dental Press Ortod Ortop Facial, 7 (6), $49-61$.

Silva Filho, O. G., Freitas, S. F. \& Cavassan, A. O. (1990). Prevalência de oclusão normal e má oclusão em escolares da Cidade de Bauru (São Paulo). Parte I: relação sagital. Rev. Odontol. Univ. São Paulo, 4 (2), 130-7.

Souza, R. S., Pinto, A. S., Shimizu, R. H., Sakima, M. T. \& Gandini Jr, LG. (2003). Avaliação do sistema de forças gerado pela alça T de retração pré-ativada segundo o padrão UNESP - Araraquara. Rev Dental Press Ortod Ortop Facial, 8 (5):113-122.

Vaden, J. L., Williams, R. A. \& Goforth, R. L. (2018). Class II correction: Extraction or nonextraction? American journal of orthodontics and dentofacial orthopedics: official publication of the American Association of Orthodontists, its constituent societies, and the American Board of Orthodontics, 154 (6), 860-876. https://doi.org/10.1016/j.ajodo.2018.06.010 